\title{
Myopathy Detection and Classification Based on the Continuous Wavelet Transform
}

\author{
Abdelali Belkhou, Abdelouahad Achmamad and Atman Jbari
}

\begin{abstract}
Electromyography (EMG) technique is often used for diagnosis of neuromuscular diseases such as myopathy that affects the muscle and causes many changes in the electromyography signal characteristics. This paper presents a new method for analysis and classification of normal and myopathy EMG signals based on the continuous wavelet transform (CWT). Classification algorithms, namely Support Vector Machine (SVM), k-Nearest Neighbor (k-NN), Decision Tree (DT), Discriminant Analysis (DA) and Native Bayes (NB) were used in our study. Five features were extracted from the CWT and employed them as input features to the classifiers. Results were evaluated and subsequently, a comparison was made in terms of performance markers, namely, accuracy, sensitivity, and specificity to ensure the efficacy of individual classifiers as well as the number and the combination of the feature sets. Results showed that k-NN classifier with an association of four features delivered the best performances with an accuracy of $93.68 \%$.
\end{abstract}

Index Terms-Electromyography (EMG), continuous wavelet transform (CWT), support vector machine (SVM), k-nearest neighbor (k-NN), decision tree (DT), discriminant analysis (DA), native bayes $(\mathrm{NB})$.

\section{INTRODUCTION}

I $\mathrm{N}$ the biomedical area, Electromyography (EMG) refers to the study of the electrical activity of the muscle [1]. From a technical side, EMG is a biological signal acquired from muscles with the aim of evaluating their activities [2]. Typically EMG is recorded by invasive and non-invasive techniques. The former employs needle electrodes and the latter employs surface electrodes to acquire signals known as intramuscular EMG and surface EMG [3]. In addition to the muscle fatigue evaluation, sports science, rehabilitation and the development of the prosthetic device purposes [4] [5], the primary use of the EMG signal is the diagnosis of the neuromuscular diseases [6] [7] [8]. As already mentioned and studied in different researches [8] [9], the most famous neuromuscular diseases of muscles are myopathy and neuropathy. In neurogenic cases, nerves of the neuromuscular system are damaged, whereas, in myopathy disease, the affected organ is the muscle itself [10].

In the literature, the diagnosis of neuromuscular diseases or more particularly, classification of EMG data into normal, myopathy, and neuropathy EMG signals, was a topic of

Manuscript received May 30, 2019; revised October 28, 2019. Date of publication November 25, 2019. Date of current version November 25, 2019 The associate editor prof. Mladen Russo has been coordinating the review of this manuscript and approved it for publication.

Authors are with the Electronic Systems, Sensors and Nanobiotechnologies Research Team, ENSET, Mohammed V University in Rabat, Morocco (e-mails: abdelali.belkhou@um5s.net.ma, abdelouahad.achmamad@um5s.net.ma, atman.jbari@um5.ac.ma).

Digital Object Identifier (DOI): 10.24138/jcomss.v15i4.796 multiple research. In [11], an accuracy of $90.7 \%$ was achieved using wavelet neural networks (WNN) based classifier with an autoregressive (AR) model of EMG signals. In [12], neurofuzzy computing techniques with autoregressive (AR), discrete wavelet transform (DWT) and wavelet packet transform (WPT) as feature extraction methods were studied. Classification accuracy of $95 \%$ was achieved. Other essential works which studied the classification of normal, myopathy, and neuropathy EMG signals can be found in [10] [13] [14]. The research and the classification into myopathic, amyotrophic lateral sclerosis (ALS) or normal EMG signals have also taken considerable importance in the last years. The authors in [15] proposed a learning scheme based on a feature fusion using multi-domain discriminant correlation analysis (MDCA) for a diagnosis of electroencephalogram (EEG) and EMG patterns. The algorithm was the object of a real-time implementation on a microcontroller device. Regarding the EMG signal diagnosis, the proposed work achieved an optimal accuracy of $98 \%$ using DA. In [16], a multiview feature fusion system is proposed. The set of features are generated in both the time and the wavelet domains. Thereafter, the discriminant correlation analysis (DCA) was performed. The proposed algorithm was tested with two EMG data sets. The authors obtained $100 \%$ in terms of accuracy, specificity and sensitivity in one of the two data sets. Other relevant multi-class studies related to myopathic, ALS and normal EMG signals can be found in [7] [17] [18] [19] [20].

Our study deals with a binary classification issue to distinguish myopathic patients from normal subjects using the CWT. Different approaches have been proposed to deal with this issue. In a recent paper [21], an autoregressive moving average (ARMA) model followed by linear discriminant analysis (LDA) algorithm was used to identify myopathic patients from normal subjects. An accuracy of $90.25 \%$ was obtained. An empirical mode decomposition (EMD) based technique for the discrimination between myopathic and normal EMG signals was proposed in [22]. In [14], the authors employed different feature techniques for the classification of EMG signals into healthy subjects or myopathic patients. The autoregressive (AR) technique along with the multilayer perceptron (MLP) classification algorithm achieved higher results with an accuracy of $83 \%$. The authors in [23] achieved $86.1 \%$ accuracy, $88.9 \%$ sensitivity and $83.3 \%$ specificity using the autocorrelation function and k-NN classifier. In another reported work [24], authors combined features extracted from tunableQ wavelet transform (TQWT) and from the time domain to classify EMG signals into normal subjects or myopathic patients. The obtained accuracy was $82.41 \%$ using random 
forest classifier.

In our previous work [25], four features were extracted from the CWT and were all fed to SVM and k-NN classifiers to detect myopathic cases from normal subjects. 10-fold crossvalidation was used as an evaluation technique. An accuracy of $91.11 \pm 0.38$ (mean \pm standard deviation) was obtained using k-NN. This work is an experimental study in which the CWT is applied along with five classification algorithms and five extracted features (latterly detailed). The main idea behind this study is the evaluation of the effect of the number and the combination of the used features on the classification accuracy. Thus, all the possible combinations of the previous features were examined to determine the most suitable parameters for our research. Besides, this work is interested in finding the association of the mother wavelet in a pretreatment step, the features, the classifier and the kernel function that ensure higher accuracy. The algorithm is tested on data which is divided into 2 separate subsets: a training set and a test set. As opposed to the 10-fold cross-validation method employed in the previous study, the included training subset signals in this work are in no way reused in the test process.

As a mathematical tool for feature extraction, the CWT is a non-stationary signal processing technique widely used in the biomedical field. Several publications proved the usefulness of CWT in EMG signal processing [10] [26] [27]. The aim of this study is to develop a new and reliable EMG classification method. In our proposed work, the CWT is applied to raw EMG data to extract useful features for classification purpose. In the classification process, feature extraction is a crucial stage, in which single scalar parameters represent the whole signal. These parameters must be meaningful as much as possible. In other words, the chosen features must have the capability to discriminate between different signals (myopathic and normal in our case). Thus, to prove the efficiency of the proposed parameters, and to get higher classification performances, our algorithm was run as much as the possible combinations of these features using five classification algorithms with different kernel functions.

The remainder of the paper is organized as follows: Section II introduces the theory of wavelet analysis and feature extraction. Section III shows the methodology used in this study. Section IV presents the experimental results of the proposed methodology with discussion and presents a comparative study with other reported works from the literature. Finally, we conclude with Section V.

\section{The Theory of Wavelet ANAlysis And Feature EXTRACTION}

\section{A. Continuous Wavelet Transform}

The CWT provides both time and frequency localization of a signal, which makes it an appropriate technique for analysing non-stationary signals such as EMG which is the subject of this study. The CWT is based on the notion of scale which is an alternative to the concept of frequency in the Fourier transform. The result of the CWT is shifted and scaled versions of the original wavelet, whereas in Fourier transform, the original signal is decomposed into sine waves of multiples frequencies [28].

The CWT, which reflects the correlation between a signal $\mathrm{x}(\mathrm{t})$ and a function known as the mother wavelet is defined by the following equations:

$$
C_{x}(\tau, \sigma)=\int_{-\infty}^{+\infty} x(t) \psi_{\tau, \sigma}^{*}(t) d t
$$

where

$$
\psi_{\tau, \sigma}(t)=\frac{1}{\sqrt{\sigma}} \psi\left(\frac{t-\tau}{\sigma}\right)
$$

$\psi(t)^{*}$ denotes the complex conjugate of the mother wavelet function $\psi(t), \sigma$ is the scale, $\tau$ refers to the translation of the wavelet and $\frac{1}{\sqrt{\sigma}}$ is used for energy normalization [2].

Generally, the choice of appropriate scales and an adequate analysing wavelet function depends on the application.

\section{B. Average Absolute Coefficient per Scale}

After we applied CWT to our EMG data, the result $C$ is an $N \times M$ coefficients matrix for each signal. $N$ reflects the number of scales whereas $M$ indicates samples of the signal. Since this representation could be difficult to interpret, we proposed in a first stage to reduce the size of the matrix to a single vector by calculating the mean absolute coefficient per scale, by the mean of the following equation:

$$
s c=\frac{\sum_{j=1}^{M}\left|C_{i j}\right|}{M} \quad i=1, \ldots, N
$$

where $s c$ is a vector which contains the average absolute coefficient per scale for one subject. The second stage of the proposed system corresponds to feature extraction and will be explained next.

\section{Feature Extraction using CWT}

Based on first observations (Fig. 2 and Fig. 3), good discrimination between normal and myopathy EMG signals could be done using the proposed algorithm presented in Fig.1.

The use of classifiers requires the extraction of the most relevant parameters in the signal. In most machine learning applications, signals are represented by their statistical information. Thus, five statistical features are extracted in our study by means of mathematical tools. The proposed features are :

- The mean scale

$$
\text { mean }_{\text {scale }}=\frac{\int_{0}^{\text {scale }_{\max }} \sigma\left|c_{x}(\sigma)\right| d \sigma}{\int_{0}^{\text {scale }_{\max }}\left|c_{x}(\sigma)\right| d \sigma}
$$

- The median scale

$$
\int_{0}^{\text {med }_{\text {scale }}}\left|c_{x}(\sigma)\right| d \sigma=\frac{1}{2} \int_{0}^{\max _{\text {scale }}}\left|c_{x}(\sigma)\right| d \sigma
$$

- The mean coefficient

$$
\text { mean }_{c w t}=\frac{\sum_{i=1}^{N} s c(i)}{N}
$$

- The minimum coefficient 


$$
\min _{c w t}=\min (s c)
$$

- The maximum coefficient

$$
\max _{c w t}=\max (s c)
$$

\section{Methodology}

\section{A. EMG Dataset}

As presented in [29], the data contains two classes: a class of normal subjects and a class of myopathic patients. The myopathic group is composed of 7 patients aged 1963 years; 5 males and 2 females. The myopathic patients had all, electrophysiological and clinical signs of myopathy. The group of the normal class contains 10 subjects, 6 males and 4 females aged 21-37 years. The normal group had no signs of neuromuscular disorders. The brachial biceps muscle was employed in this work because it is one of the most investigated muscles in the EMG analysis.

The EMG signals were recorded under usual conditions for Motor Unit Action Potential (MUAP) analysis. The recordings were made at a low voluntary and constant level of contraction. A standard concentric needle electrode was used. The signals were recorded from five places in the muscle at three levels of insertion (deep, medium, low). The signals were recorded at a sampling frequency of $23437 \mathrm{~Hz}$. The high and low pass filters of the EMG amplifier were set at $2 \mathrm{~Hz}$ and $10 \mathrm{kHz}$ [29].

\section{B. Classification Process}

The EMG data used in this study is divided into a training data set and test data set. The training data represents $75 \%$ of the total data set and it is used to build the classification model. The test data which represents $25 \%$ of the total data is used for validation purpose. The distribution of records in the two data sets for each class is shown in Table I.

TABLE I

DistRIBUTION OF RECORDS IN THE TwO DATASETS

\begin{tabular}{llll}
\hline Class & Training set & Test set & Total \\
\hline Normal & 201 & 68 & 269 \\
Myopathy & 80 & 27 & 107 \\
\hline
\end{tabular}

After extracting the previously mentioned features, the next step is the classification of all signals into two groups: normal subjects and myopathic patients. In our study, five supervised learning classifiers are applied. Table II summarizes the different classifiers and kernels used. Regarding the k-NN classifier, it is evaluated via different $\mathrm{k}$ parameter values.

Next, classification results are examined. Measurements such as accuracy, sensitivity, and specificity are used and defined as follows [11]:

- Specificity (Spec): refers to the number of correctly classified normal subjects divided by the total number of normal subjects.

- Sensitivity (Sens): refers to the number of correctly classified myopathic subjects divided by the total number of myopathic subjects

- Accuracy (Acc): refers to the number of correctly classified subjects divided by the total number of subjects.
These measurements are calculated by the following equations [30]:

$$
\begin{gathered}
\text { Sensitivity }=\frac{T_{p}}{T_{p}+F_{N}} \times 100 \\
\text { Specificity }=\frac{T_{N}}{T_{N}+F_{P}} \times 100 \\
\text { Accuracy }=\frac{T_{N}+T_{P}}{T_{N}+T_{P}+F_{N}+F_{P}} \times 100
\end{gathered}
$$

where $T_{P}$ is the number of true positives, $T_{N}$ true negatives, $F_{P}$ false positives and $F_{N}$ false negatives [31].

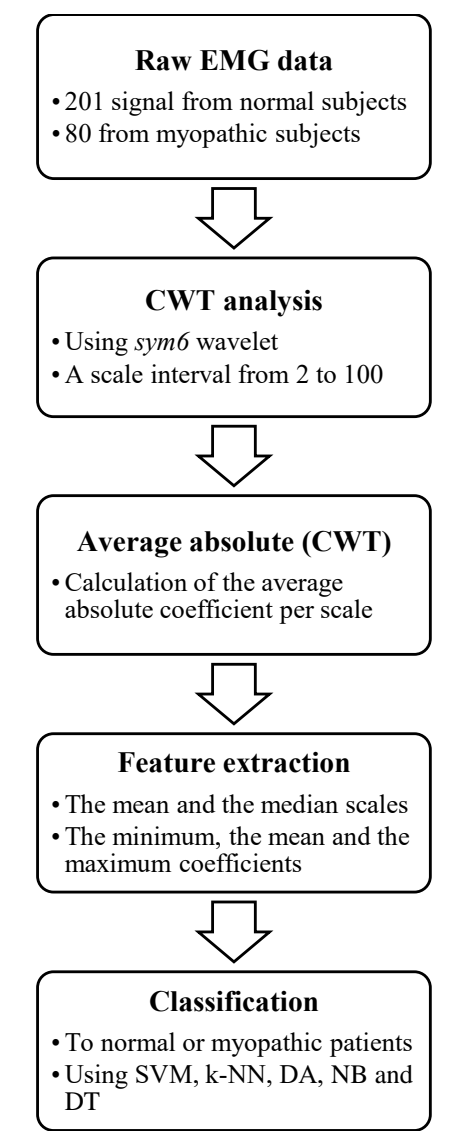

Fig. 1. Block diagram of the proposed work.

\section{EXPERIMENTAL RESULTS AND DISCUSSION}

This paper presents a new technique for EMG signal analysis and classification (Fig. 1). The study concerns two groups: normal subjects and myopathic patients. An example of a typical EMG signal for each group is given in Fig. 2.

In our study, the CWT is used to analyse EMG signals. In order to determine the suitable CWT parameters (the mother wavelet and the scales), a pretreatment process is done. Wavelet functions such as "db4", "sym6" and "haar" wavelets were tested. Best performances and low computation time were obtained via "sym6". Following this result, the whole study is completed using "sym6" wavelet function. 
TABLE II

Classifiers AND KERNELS USED IN THIS STUdY

\begin{tabular}{l|lllrr}
\hline Classifier & SVM & DA & DT & NB & k-NN \\
\hline \multirow{3}{*}{ Kernel/ K value } & Linear(Lin) & Linear(Lin) & Exact(Ex) & Normal(Nor) & 3 \\
& Polynomial(Pol) & PseudoQuadratic(PsQ) & PullLeft(Pul) & Triangle(Tri) & 5 \\
& RBF & DiagLinear(DiagL) & PCA & Epanechnikov(Ep) & 7 \\
& - & PseudoLinear(PsL) & - & Box & 9 \\
\hline
\end{tabular}

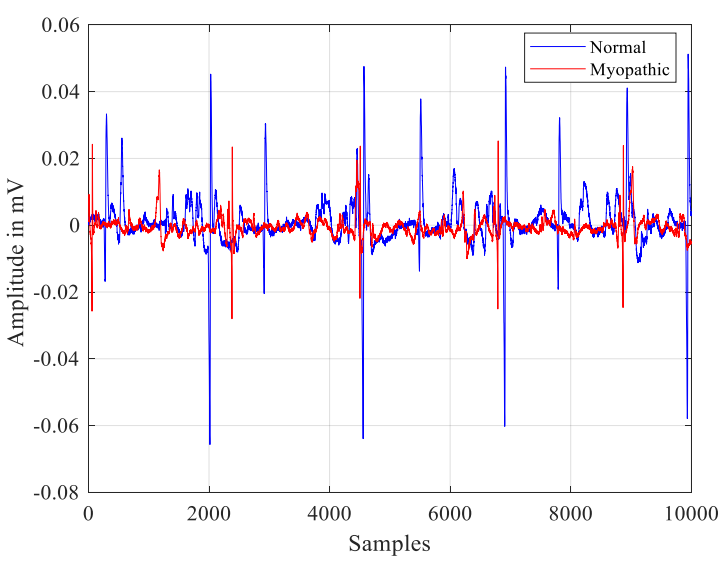

Fig. 2. Typical normal and myopathic EMG signals.

Table III summarizes the empirically obtained CWT parameters.

TABLE III

CWT PARAMETERS USED IN THIS STUDY

\begin{tabular}{lll}
\hline Mother wavelet & Scale interval & $\begin{array}{l}\text { Corresponding frequency } \\
\text { interval }(\mathrm{Hz})\end{array}$ \\
\hline Sym6 & $2-100$ & $170-8523$ \\
\hline
\end{tabular}

After CWT is applied to raw EMG data, and to correctly identify myopathic patients from normal subjects, we followed a procedure based on the calculation of the mean absolute CWT coefficient per scale (Fig. 3). Meaningful features were then proposed to be extracted for classification purpose.

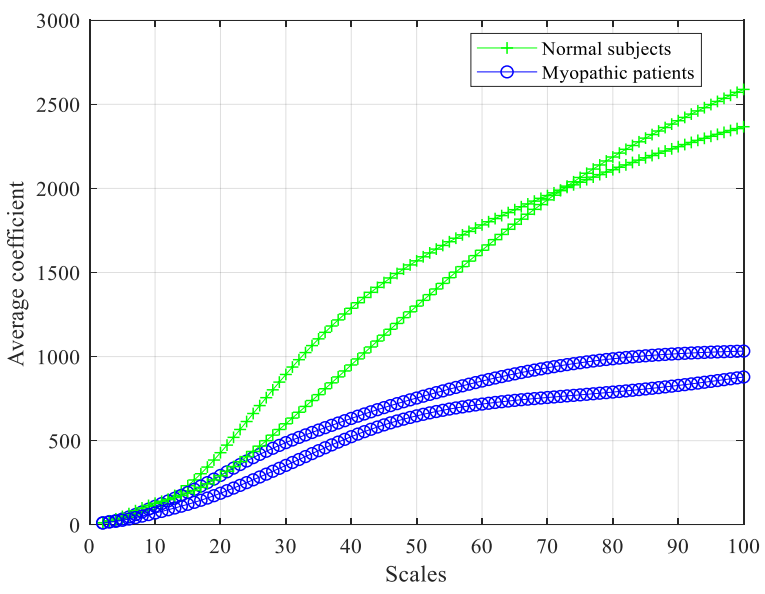

Fig. 3. The average coefficient evolution per scale for four significant subjects: two normal and two myopathic.
A meaningful difference between normal and myopathic subjects based on time scale analysis of the EMG signal is shown in Fig. 3. The evolution of the mean coefficient per scale of normal subjects is more significant than myopathic ones. The box-and-whisker plot of this result is illustrated in Fig. 4. As shown in Fig. 3 and Fig. 4, this technique has efficiency in the discrimination between myopathy patients and normal subjects. In order to classify all signals, five features are extracted: the mean and the median scales, the minimum, the mean and the maximum coefficients.

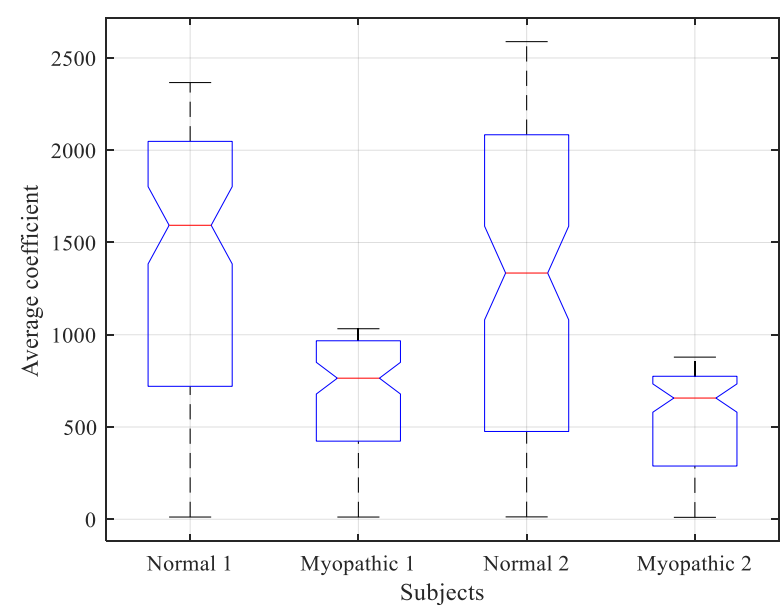

Fig. 4. The box-and-whisker plot of the average coefficient evolution per scale for four significant subjects: two normal and two myopathic.

A correct diagnosis of myopathic cases requires a higher classification accuracy. Thus, our algorithm was run several times to find combinations of features and kernels that ensure the best results.

Table IV shows the best results obtained with the corresponding kernel functions using all the five parameters previously presented.

TABLE IV

Classification Result Using all FEATURES

\begin{tabular}{lllll}
\hline Classifier & Acc $(\%)$ & Sens $(\%)$ & Spec $(\%)$ & Kernel / K value \\
\hline SVM & 91.58 & 81.48 & 95.59 & RBF \\
DA & 90.53 & 85.18 & 92.65 & Lin \\
NB & 89.47 & 88.89 & 89.71 & Ep - Tri \\
k-NN & 90.53 & 88.89 & 91.18 & 9 \\
DT & 82.10 & 92.59 & 77.94 & all kernels \\
\hline
\end{tabular}

These results show that SVM classifier achieved the best performance with an accuracy of $91.58 \%$, a sensitivity of $81.48 \%$ and a specificity of $95.59 \%$ using RBF kernel. 
Regarding the other classifiers performances, accuracies of $90.53 \%, 89.47 \%$, and $82.10 \%$ are obtained using $\mathrm{k}-\mathrm{NN}$ and DA classifiers, NB, and DT, respectively.

To get higher accuracies, we propose to run our algorithm as much as the possible combinations of features. Table $\mathrm{V}$ summarizes the results of the best performances combination of only four features. The best combination found is compound from the following features: The mean and the median scales, the mean and the minimum coefficients.

Using only four features, the $\mathrm{k}-\mathrm{NN}$ classifier with $\mathrm{k}=7$ demonstrated the highest performance with an accuracy of $93.68 \%$, a sensitivity of $88.89 \%$ and a specificity of $95.59 \%$. SVM classifier with the RBF kernel performed the secondhighest classification accuracy of $92.63 \%$. On the one side, DA and NB classifiers kept almost the same performances as previously when all five features were used. On the other side, DT classifier achieved an accuracy of $83.16 \%$ using all kernels. As compared to the case with five features, results become higher using only four.

TABLE V

Classification Result Using Best Four Combined Features

\begin{tabular}{lllll}
\hline Classifier & Acc $(\%)$ & Sens $(\%)$ & Spec $(\%)$ & Kernel / K value \\
\hline SVM & 92.63 & 85.18 & 95.59 & RBF \\
DA & 90.53 & 88.89 & 91.18 & PsQ \\
NB & 89.47 & 85.18 & 91.18 & Nor - Tri \\
k-NN & 93.68 & 88.89 & 95.59 & 7 \\
DT & 83.16 & 88.89 & 80.88 & all kernels \\
\hline
\end{tabular}

Table VI presents the results of the best three combined features, which are: the mean scale, the minimum, and the maximum coefficients.

TABLE VI

Classification Result Using Best Three Combined Features

\begin{tabular}{lllll}
\hline Classifier & Acc $(\%)$ & Sens $(\%)$ & Spec $(\%)$ & Kernel / K value \\
\hline SVM & 90.53 & 81.48 & 94.12 & RBF \\
DA & 89.47 & 88.89 & 89.71 & Lin \\
NB & 88.42 & 88.89 & 88.23 & Box \\
k-NN & 91.58 & 85.18 & 94.12 & $5-7$ \\
DT & 81.05 & 85.18 & 79.41 & all kernels \\
\hline
\end{tabular}

As it is clear in the table above, stability in the overall performances is noticed. All classifiers except DT have an accuracy higher than $88 \%$, while DT achieved only $81 \%$. In general, the specificity values are higher than sensitivity values for all classifiers except for DT classifier.

Table VII illustrates a comparison between the performances of the five classifiers using only two features. The best two combined features are the mean scale and the minimum coefficient.

We can notice from the results of Table VII that the overall performances decreased for the majority of classifiers except for SVM and NB classifiers. An accuracy of $90.53 \%$ is achieved with the Linear kernel function of the SVM. The obtained sensitivity and specificities are $88.89 \%$ and $91.18 \%$ respectively. A significant decrease is to be noticed in DT
TABLE VII

Classification Result Using Best Two Combined Features

\begin{tabular}{lllll}
\hline Classifier & Acc $(\%)$ & Sens $(\%)$ & Spec $(\%)$ & Kernel / K value \\
\hline SVM & 90.53 & 88.89 & 91.18 & Lin \\
DA & 89.47 & 81.48 & 92.65 & DiagL \\
NB & 89.47 & 77.78 & 94.12 & Box \\
k-NN & 89.47 & 85.18 & 91.18 & 7 \\
DT & 76.84 & 70.37 & 79.41 & all kernels \\
\hline
\end{tabular}

results. The DT accuracy is only $76.84 \%$, while the sensitivity and the specificities are $70.37 \%$ and $79.41 \%$ respectively. Table VIII shows the obtained results with the corresponding kernel function using only one parameter. Best results were obtained using the mean scale feature.

TABLE VIII

Classification Result Using Only One Feature

\begin{tabular}{lllll}
\hline Classifier & Acc $(\%)$ & Sens $(\%)$ & Spec $(\%)$ & Kernel / K value \\
\hline SVM & 84.21 & 70.37 & 89.71 & Lin-RBF \\
DA & 85.26 & 74.07 & 89.71 & all kernels \\
NB & 84.21 & 70.37 & 89.71 & all kernels \\
k-NN & 81.05 & 74.07 & 83.82 & 7 \\
DT & 73.68 & 59.26 & 79.41 & all kernels \\
\hline
\end{tabular}

Using only the mean scale as a feature, the best-obtained accuracy is $85.26 \%$ and was obtained via all kernels of DA classifier. DT had the lowest result with an accuracy of $73.68 \%$. The following figure presents the evolution of the accuracy of all classifier along with the number of the used features.

From the obtained results, and as the Fig. 5 shows, it is noticed that generally, the accuracy significantly increases with an increase in the number of features used to train the classifiers. The highest results of all classifiers were obtained using a combination of the following four features: the mean and the median scales, the mean and the minimum coefficients. The best-obtained accuracy was $93.68 \%$ and was achieved using $\mathrm{k}-\mathrm{NN}$ classifier with $\mathrm{k}=7$. SVM achieved an accuracy of $92.63 \%$ using the Polynomial kernel. These results show the effectiveness of these two classifiers compared to others (DT in particular), the thing that goes with the previous results from the literature [7] [25] [31].

The summary of all best results for each classifier with the specific kernel and the best features combination employed is presented in Table IX.

The findings are of a higher accuracy when compared with other studies which investigated the normal and the myopathic EMG classes (Table X).

\section{CONCLUSION}

In this work, a new approach based on CWT to analyse and classify EMG data into two groups: normal and myopathy, is proposed. The CWT was performed, and then five features that we applied at a later stage as inputs to classifiers were extracted. The suggested parameters in this study are the 
TABLE IX

Summary of the Best Classification Results of all Classifiers with the Features Combination Used

\begin{tabular}{llllll}
\hline Classifier & Acc $(\%)$ & Sens $(\%)$ & Spec $(\%)$ & Kernel / K value & Features combination \\
\hline k-NN & 93.68 & 88.89 & 95.59 & 7 & mean and median scales - mean and minimum coefficients \\
SVM & 92.63 & 85.18 & 95.59 & RBF & mean and median scales - mean and minimum coefficients \\
DA & 90.53 & 88.89 & 91.18 & PsQ & mean and median scales - mean and minimum coefficients \\
NB & 89.47 & 88.89 & 89.71 & Ep - Tri & all features \\
DT & 83.16 & 88.89 & 80.88 & PCA-Exact-PullLeft & mean and median scales - mean and minimum coefficients \\
\hline
\end{tabular}

TABLE X

COMParison of the Proposed Work With Other Reported Works Which STUdied Two-Class Classification: MYOPATHIC AND NORMal Classes

\begin{tabular}{llll}
\hline Work & Methods and classification algorithms used & Acc $(\%)$ & Sens $(\%)$ \\
\hline Fattah et al. [23] & Auto-correlation - k-NN & 86.1 & 88.9 \\
Elamvazuthal et al. [14] & Auto-regressive - MULTI-LAYER PERCEPTRON & 83 & - \\
Joshi et al. [24] & Tunable-Q wavelet - RANDOM FOREST & 81.14 & - \\
Lahmiri and Boukadoum [21] & Full ARMA - LDA & 90.25 & - \\
Proposed work & CWT - k-NN & 93.68 & 83.67 \\
\hline
\end{tabular}

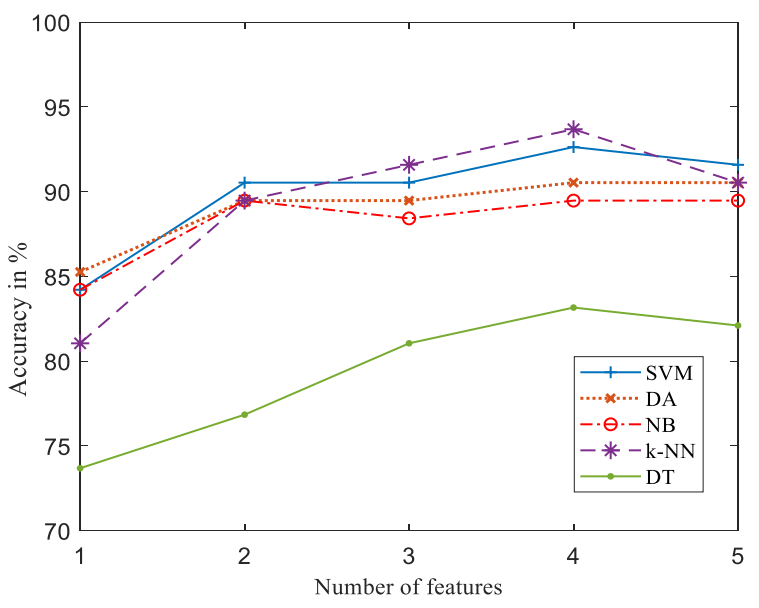

Fig. 5. Evolution of the accuracy of all classifier following the number of the used features.

mean and the median scales, the mean, the minimum and the maximum coefficients. Five classification algorithms were also used: Support Vector Machine (SVM), Discriminant Analysis (DA), Native Bayes (NB), k-Nearest Neighbour (k-NN) and Decision Tree (DT). For each classification algorithm, multiple kernel functions were explored. The core focus of this research is to investigate the highest classification performances by evaluating the impact of the number and the association of the proposed features on the classification results. From the research that has been carried out, it can be concluded that our proposed technique using the CWT is an efficient tool for the diagnosis of myopathy disease. This binary classification algorithm delivered consistent results in discrimination between myopathic patients and normal subjects using intramuscular EMG signals. The findings of our research are quite convincing, and thus, this technique can be generalised in the future as a multi-class classification method. The proposed work can be applied and also tested for other biomedical signals.

\section{REFERENCES}

[1] D. Yang, H. Zhang, Y. Gu, and H. Liu, "Accurate EMG onset detection in pathological , weak and noisy myoelectric signals," Biomed. Signal Process. Control, vol. 33, pp. 306-315, 2017. DOI: 10.1016/j.bspc.2016.12.014.

[2] A. Belkhou, A. Jbari, and L. Belarbi, "A continuous wavelet based technique for the analysis of electromyography signals," International Conference on Electrical and Information Technologies (ICEIT), Rabat, pp. 1-5, 2017. DOI: 10.1109/EITech.2017.8255232.

[3] M. Cifrek, V. Medved, S. Tonković, and S. Ostojić, "Surface EMG based muscle fatigue evaluation in biomechanics," Clin. Biomech., vol. 24, no. 4, pp. 327-340, 2009. DOI : 10.1016/j.clinbiomech.2009.01.010.

[4] V. K. Mishra, V. Bajaj, A. Kumar, and G. K. Singh, "Analysis of ALS and normal EMG signals based on empirical mode decomposition," IET Sci. Meas. Technol., vol. 10, no. 8, pp. 963-971, 2016. DOI: 10.1049/ietsmt.2016.0208.

[5] G. R. Naik, D. K. Kumar and M. Palaniswami, "Classification of low level surface electromyogram using independent component analysis," in IET Signal Processing, vol. 4, no. 5, pp. 479-487, Oct. 2010. DOI 10.1049/iet-spr.2007.0211.

[6] V. K. Mishra, V. Bajaj, A. Kumar, D. Sharma, and G. K. Singh, "An efficient method for analysis of EMG signals using improved empirical mode decomposition," AEU - Int. J. Electron. Commun., vol. 72, pp. 200-209, 2017. DOI: 10.1016/j.aeue.2016.12.008.

[7] E. Gokgoz and A. Subasi, "Effect of multiscale PCA de-noising on EMG signal classification for diagnosis of neuromuscular disorders," J. Med. Syst., vol. 38, no. 4, 2014. DOI: 10.1007/s10916-014-0031-3.

[8] A. Goen, "Classification of EMG Signals for Assessment of Neuromuscular Disorders," Int. J. Electron. Electr. Eng., vol. 2, no. 3, pp. 242-248, 2014. DOI: $10.12720 /$ ijeee.2.3.242-248.

[9] C. D. Katsis, Y. Goletsis, A. Likas, D. I. Fotiadis, and I. Sarmas, "A novel method for automated EMG decomposition and MUAP classification," Artif. Intell. Med., vol. 37, no. 1, pp. 55-64, 2006. DOI: 10.1016/j.artmed.2005.09.002.

[10] R. Istenič, P. A. Kaplanis, , C. S. Pattichis, et al., "Multiscale entropybased approach to automated surface EMG classification of neuromuscular disorders," Med. Biol. Eng. Comput, vol. 48, no. 8, pp. 773-781, 2010. DOI: $10.1007 / \mathrm{s} 11517-010-0629-7$.

[11] A. Subasi, M. Yilmaz, and H. R. Ozcalik, "Classification of EMG signals using wavelet neural network," J. Neurosci. Methods, vol. 156, no. 1-2, pp. 360-367, 2006. DOI: 10.1016/j.jneumeth.2006.03.004.

[12] A. Subasi, "Classification of EMG signals using combined features and soft computing techniques," Appl. Soft Comput. J., vol. 12, no. 8, pp. 2188-2198, 2012. DOI:10.1016/j.asoc.2012.03.035.

[13] C. D. Katsis, T. P. Exarchos, C. Papaloukas, Y. Goletsis, D. I. Fotiadis, and I. Sarmas, "A two-stage method for MUAP classification based on EMG decomposition," Comput. Biol. Med., vol. 37, no. 9, pp. 1232-1240, 2007. DOI: 10.1016/j.compbiomed.2006.11.010. 
[14] I. Elamvazuthi, N. H. X. Duy, Z. Ali, S. W. Su, M. K. A. A Khan, and S. Parasuraman, "Electromyography (EMG) based Classification of Neuromuscular Disorders using Multi-Layer Perceptron,' Procedia Comput. Sci., vol. 76, no. Iris, pp. 223-228, 2015. DOI: 10.1016/j.procs.2015.12.346.

[15] A. Hazarika, L. Dutta, P. Barman, A. Subasi, M. Bhuyan and C. Talukdar, "Real-time Implementation of a Multi-domain Feature Fusion Model Using Inherently Available Large Sensor Data," in IEEE Transactions on Industrial Informatics. DOI: 10.1109/TII.2019.2914975.

[16] A. Hazarika, L. Dutta, M. Barthakur, and M. Bhuyan, "A Multiview Discriminant Feature Fusion-Based Nonlinear Process Assessment and Diagnosis: Application to Medical Diagnosis," IEEE Trans. Instrum. Meas., vol. 68, no. 7, pp. 2498-2506, 2019. DOI: 10.1109/TIM.2018.2866744.

[17] E. Gokgoz and A. Subasi, "Comparison of decision tree algorithms for EMG signal classification using DWT," Biomed. Signal Process. Control, vol. 18, pp. 138-144, 2015. DOI: 10.1016/j.bspc.2014.12.005.

[18] V. K. Mishra, V. Bajaj, and A. Kumar, "Classification of normal, ALS, and myopathy EMG signals using ELM classifier," Proceeding IEEE - 2nd Int. Conf. Adv. Electr. Electron. Information, Commun. BioInformatics, IEEE - AEEICB, pp. 455-459, 2016. DOI: 10.1109/AEEICB.2016.7538330.

[19] A. Hazarika, L. Dutta, M. Barthakur, and M. Bhuyan, "Fusion of projected feature for classification of EMG patterns," Int. Conf. Access. to Digit. World, ICADW 2016 - Proc., pp. 69-74, 2017. DOI: 10.1109/ICADW.2016.7942515.

[20] A. Hazarika, L. Dutta, M. Boro, M. Barthakur, and M. Bhuyan, "An automatic feature extraction and fusion model: application to electromyogram (EMG) signal classification," Int. J. Multimed. Inf. Retr. vol. 7, no. 3, pp. 173-186, 2018. DOI: 10.1007/s13735-018-0149-z.

[21] S. Lahmiri, M. Boukadoum, "Improved Electromyography Signal Modeling for Myopathy Detection," IEEE International Symposium on Circuits and Systems (ISCAS), Florence, pp. 1-4, 2018. DOI: 10.1109/ISCAS.2018.8350893.

[22] V. K. Mishra, V. Bajaj, A. Kumar, and D. Sharma, "Discrimination between Myopathy and normal EMG signals using intrinsic mode functions," Int. Conf. Commun. Signal Process. ICCSP, pp. 299-303, 2016. DOI: 10.1109/ICCSP.2016.7754143.

23] S. A. Fattah, A. B. M. S. U. Doulah, and M. Ahmed, "Evaluation of Different Time and Frequency Domain Features of Motor Neuron and Musculoskeletal Diseases," International Journal of Computer Applications, vol. 43, no. 23, pp. 34-40, 2012. DOI: 10.5120/6422-8922.

[24] D. Joshi, A. Tripathi, R. Sharma and R. B. Pachori, "Computer aided detection of abnormal EMG signals based on tunable-Q wavelet transform," 2017 4th International Conference on Signal Processing and Integrated Networks (SPIN), Noida, pp. 544-549, 2017. DOI: 10.1109/SPIN.2017.8050010.

[25] A. Belkhou, A. Achmamad, A. Jbari, "Classification and Diagnosis of Myopathy EMG Signals Using the Continuous Wavelet Transform," 2019 Scientific Meeting on Electrical-Electronics and Biomedical Engineering and Computer Science (EBBT), Istanbul, Turkey, pp. 1-4, 2019 DOI: 10.1109/EBBT.2019.8742051.

[26] M. Yochum, T. Bakir, S. Binczak, R. Lepers, "EMG artifacts removal during electrical stimulation, a CWT based technique," 2014 IEEE REGION 10 SYMPOSIUM, Kuala Lumpur, pp. 137-140, 2014. DOI: 10.1109/TENCONSpring.2014.6863012.

[27] R. N. Leao and J. A. Burne, "Continuous wavelet transform in the evaluation of stretch reflex responses from surface EMG," J Neurosci Methods, vol. 133, no. 1-2, pp. 115-125, 2004. DOI: 10.1016/j.jneumeth.2003.10.003.

[28] H. Khorrami and M. Moavenian, "A comparative study of DWT, CWT and DCT transformations in ECG arrhythmias classification," Expert Syst. Appl., vol. 37, no. 8, pp. 5751-5757, 2010. DOI: 10.1016/j.eswa.2010.02.033

[29] M. Nikolic, "Detailed Analysis of Clinical Electromyography Signals EMG Decomposition, Findings and Firing Pattern Analysis in Controls and Patients with Myopathy and Amytrophic Lateral Sclerosis," PhD Thesis, Faculty of Health Science, University of Copenhagen, 2001.

[30] R. R. Sharma and R. B. Pachori, "Time-frequency representation using IEVDHM-HT with application to classification of epileptic EEG signals," IET Sci. Meas. Technol., vol. 12, no. 1, pp. 72-82, 2018. DOI: 10.1049/iet-smt.2017.0058

[31] A. Benba, A. Jilbab, and A. Hammouch, "Discriminating Between Patients With Parkinson's and Neurological Diseases Using Cepstral Analysis," IEEE Trans. Neural Syst. Rehabil. Eng., vol. 24, no. 10, pp. 1100-1108, 2016. DOI: 10.1109/TNSRE.2016.2533582.

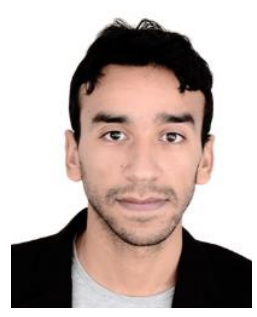

A. Belkhou received his engineer degree in Electrical Engineering in 2016 at Cadi Ayyad University in Marrakech. He is currently a $\mathrm{PhD}$ student with the Research Team of Electronic Systems, Sensors and Nano-biotechnologies (E2SN) at ENSET, Mohammed V University in Rabat, Morocco. His current research interests include electronics and signal processing.

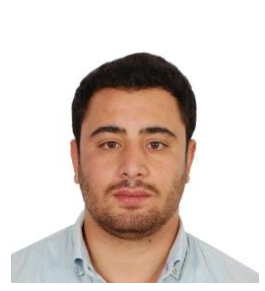

A. Achmamad was born in Rif area of Morocco, at 27 th January 1992. He received his master degree in Electrical Engineering in 2017 from High School of Technical Education, Rabat. Currently PhD student with the Research Team of Electronic Systems, Sensors and Nano-biotechnologies (E2SN) at EN SET, Mohammed V University in Rabat, Morocco. $\mathrm{He}$ is interested in the biomedical field, especially biological signal processing.

A. Jbari is currently a Professor at the Electrical Engineering Department of ENSET "Ecole Normale Supérieure de l'Enseignement Technique", Mohammed V University in Rabat, Morocco. In 2009, he received his $\mathrm{PhD}$ degree in Compute and Telecommunications from Mohammed V-Agdal University. His current research interests include signal processing, blind source separation and embedded electronic systems. He is a member of Electronic Systems, Sensors and Nano-biotechnologies research group. 